\title{
ABOUT THE EXISTENCE AND UNIQUENESS THEOREM FOR HYPERBOLIC EQUATION
}

\author{
M. E. KHALIFA \\ Department of Mathematics \\ Faculty of Science \\ Benha University \\ Benha, Egypt \\ (Received August 29, 1989 and in revised form Apri1 17, 1992)
}

ABSTRACT. In this paper we prove the existence and uniqueness theorem for almost everywhere solution of the hyperbolic equation using the method of successive approximations [1].

KEY WORDS AND PHRASES. Hyperbolic equation, existence and uniqueness.

1991 AMS SUBJECT CLASSIFICATION CODE. 35H05.

\section{INTRODUCTION.}

Mixed problems for partial differential equations have been investigated by a number of authors [2], [3], [4], [5]. In this case we investigate the almost everywhere solution for the hyperbolic equation that have been studied in [6]. Namely, the solution for the hyperbolic equation in the space $B_{2,2, T}^{2,1}$ with a nonlinear operator at the right hand side.

2. STATEMENT OF THE PROBLEM.

Consider the following system

$$
u_{t t}(t, x)-L u(t, x)=F(u(t, x)) \quad \text { in } Q_{T}
$$

subject to the initial conditions

$$
u(0, x)=\varnothing(x) \quad u_{t}(0, x)=\psi(x) \quad x \in \Omega,
$$

and the boundary condition

$$
u(t, x) \mid \Gamma=0 \quad t \in[0, T]
$$

where $Q_{T}=[0, T] \times \Omega, 0<T<\infty, \Omega$ is a bounded domain in $R^{n}$ and $G$ is the boundary of $O$;

$$
L(u)=\sum_{i, j=1}^{n} \frac{\partial}{\partial x_{i}}\left(a_{i j}(x) \frac{\partial u}{\partial x_{j}}\right)-a(x) u
$$

and moreover the functions $a_{i j}(x)$ have continuous $\bar{\Omega}$ and $\frac{\partial a_{i j}(x)}{\partial x_{k}}, a(x)$ are measurable and bounded in $\Omega$ and satisfy the following conditions in $\Omega$ :

$$
a_{i j}(x)=a_{j i}(x), \quad a(x) \geq 0, \sum_{i, j=1}^{n} a_{i j}(x) \xi_{i} \xi_{j} \geq a \sum_{i=1}^{n} \xi_{i}^{2},
$$

$\xi_{i}$ are any real number; $\psi(\xi), \varphi(x)$ are given functions in $\Omega ; F$ is a nonlinear operator.

3. PRELIMINARIES. 
DEFINITION 1. The almost everywhere solution for the problem (2.1)-(2.3) is the function $u(x, t)$, element of $w_{2}^{2}\left(Q_{T}\right)$, belongs to $D_{1}^{0}\left(Q_{T}\right)$ and satisfies (2.1) almost everywhere in $Q_{T}$ and $t \rightarrow+0$ satisfies the following

$$
\int_{\Omega}[u(t, x)-\varnothing(x)]^{2} d x=0, \quad \int_{\Omega}\left[\frac{\partial u(t, x)}{\partial t}-\psi(x)\right]^{2} d x=0
$$

DEFINITION 2. We define the space $B_{\beta_{0}, \ldots, \beta_{\ell}, T}^{\alpha_{0}, \ldots, \alpha}$ of all functions $u(t, x)=\sum_{s=1}^{\infty} u_{s}(t) \vartheta_{s}(x)$ in $Q_{T}=[0, T] \times \Omega$, where $v_{s}(x)$ are eigenfunctions for the operator $L$ with the boundary condition (2.3) corresponding to the eigenvalues $\lambda_{s}$

$$
\left(0<\lambda_{s} \rightarrow \text { as } s \rightarrow \infty\right)[7], \quad u_{s}(t) \text { are } \ell \geq 0
$$

times continuously differentiable in $[0, T]$ and

$$
\sum_{i=1}^{l}\left\{\sum_{s=1}^{\infty}\left[\lambda_{s}^{\alpha_{i}} \max _{0 \leq t \leq T}\left|u_{s}^{(i)}(t)\right|\right]^{\beta_{i}}\right\}^{1 / \beta_{i}}<+\infty
$$

and has the norm

$$
\|u\|_{B_{\beta_{0}, \ldots, \beta_{\ell}, T}^{\alpha_{0}, \ldots, \alpha_{\ell}}}=\sum_{i=1}^{\ell}\left\{\sum_{s=1}^{\infty}\left[\lambda_{s}^{\alpha_{i}} \max _{0 \leq t \leq T}\left|u_{s}^{(i)}(t)\right|\right]^{\beta_{i}}\right\}^{1 / \beta_{i}}
$$

where $\alpha_{i} \geq 0,1 \leq \beta_{i} \leq 2,(i=0, \ldots, \ell)$.

DEFINITION 3. The function $u_{s}(t)$ is called the $s$-component of the function

$$
u,(t, x)=\sum_{s=1}^{\infty} u_{s}(t) \ell_{s}(x)
$$

and $\mu_{x}(s=1,2, \ldots)$ is the set of all $s$-components of elements of $\mu$ where $\mu \subset B_{\beta_{0}, \ldots, \beta_{\ell}, T}^{\alpha_{0}, \ldots, \alpha} \ell$

(a) for every $s(s=1,2, \ldots)$ the set $\mu$ is compact in $C^{\ell}[0, T]$; and

THEOREM 2.1. The necessary and sufficient conditions for $\mu$ to be compact in $B_{\beta_{0}, \ldots, \beta_{\ell}, T}^{\alpha_{0}, \ldots, \alpha}$

(b) for any given $\epsilon>0$ there exists a natural number $n_{\epsilon}$ so that for all $u(t, x)=\sum_{s=1}^{\infty} u_{s} \ell_{s}(x) \epsilon \mu$,

$$
\sum_{i=1}^{\ell}\left\{\sum_{s=n_{\epsilon}}^{\infty}\left[\lambda_{s}^{\alpha_{i}} \max _{0 \leq t \leq T}\left|u_{s}^{(i)}(t)\right|\right]^{\beta_{i}}\right\}^{1 / \beta_{i}}<\epsilon .
$$

This theorem can be proved analogously as in ([9] page 277-278).

LEMMA 1. For any almost everywhere solution $u(t, x)$ of $(2.1)-(2.3)$ functions $u_{s}(t)=\int_{\Omega} u(t, x) \ell_{s}(x) d x$ satisfy the following system $([7],[8])$

$$
\begin{aligned}
u_{s}(t)=\emptyset_{s} \cos \lambda_{s} t+\frac{\psi_{s}}{\lambda_{s}} \sin \lambda_{s} t+ \\
\frac{1}{\lambda_{s}} \int_{0}^{t} \int_{\Omega} F(u(\tau, x)) \cdot \ell_{s}(x) \sin \lambda_{s}(t-\tau) d x d \tau,(s=1,2, \ldots),
\end{aligned}
$$


where

$$
\phi_{s}=\int_{\Omega} \psi(x) \psi_{s}(x) d x, \quad \psi_{s}=\int_{\Omega} \imath \cdot(x) \psi_{s}(x) d x .
$$

\section{ASSUMPTION AND RESULTS.}

THEOREM 3.1. Let

1. $a_{1}(x)$ are continuously differentiable on $\bar{\Omega}$ and $a(x)$ continuous on $\bar{\Omega}$;

2. The eigcifunctions $v_{s}$ ire twice continuously differentiable on $\bar{\Omega}$ :

3. $\phi(x) \in W_{2}^{2}(\Omega) \cap D^{o}(\Omega) . \quad(x) \in D^{o}(\Omega)$;

4. $F: B_{2,2, T}^{2,1} \cup\left(W_{2}^{2}\left(Q_{T}\right) \cap B_{2,2, T}^{1,0}\right) \rightarrow W_{x, t, 2}^{1,0}\left(Q_{T}\right)$ and satisfies

for all $u \in B_{2,2, T}^{2,1}$; where $c(t), d(t) \in L_{2}(0, T)$.

$$
\|F(u(t, x))\|_{W_{2}^{1}(\Omega)} \leq c(t)+d(t)\|u\|{ }_{B_{2,2, t}^{2,1}}
$$

5. For any $u, v \in \mathscr{K}_{o}$ (where $\mathscr{K}_{o}$ is the sphere $\|u\|_{B_{2,2, T}^{2,1}} \leq C_{o}$ )

$$
\| F(u, t, x))-F\left(v(t, x)\left\|W_{2}^{1}{ }^{(\Omega)} \leq g(t)\right\| u-v \|_{B_{2,2, t}^{2,1},} g(t) \in L_{2}(0, T),\right.
$$

where

$$
C_{o}=\left\{\left[2\|W(t, x)\|_{B_{2,2, T}^{2,1}}^{2}+16 T a_{o}^{2}\|c(t)\|_{L_{2}(0, T)}^{2}\right] \exp \left[16 T a_{o}^{2}\|d(t)\|_{L_{2}(0, T)}^{2}\right]\right\}^{1 / 2}
$$

and

$$
a_{o}^{2}=\max \left\{n \cdot \max _{\imath \jmath}\left\{\left\|a_{i j}(x)\right\|_{C(\bar{\Omega})}\right\},\|a(x)\|_{C(\bar{\Omega})}\right\}
$$

6. For any $u \in B_{2,2 T}^{2,1} \cup\left(W_{2}^{2}\left(Q_{T}\right) \cap B_{2,2, T}^{1,0}\right)$ and $t \in[0, T] F(u(t, x)) \in D^{o}(\Omega)$.

Then the problem (2.1) - (2.3) has a unique solution,

PROOF. Let

$$
W(x, t)=\sum_{s=1}^{\infty}\left(\phi_{s} \cos \lambda_{s} t+\frac{\psi_{s}}{\lambda_{s}} \sin \lambda_{s} t\right) \vartheta_{s}(x),
$$

and

$$
P F(u)=\sum_{s=1}^{\infty} \frac{1}{\lambda_{s}} \int_{0}^{t} \int_{\Omega} F(u(\tau, x)) \cdot \vartheta_{s}(x) \sin \lambda_{s}(t-\tau) d x d \tau \cdot \vartheta_{s}(x)
$$

From (3.4) and (3.5) let us assume that

$$
Q(u)=W+P F(u)
$$

Then it is easy to see that the operator $Q$ acts in $B_{2,2, T}^{2,1}$ and satisfies Lipschitz condition

$$
\|Q(u)-Q(v)\|_{B_{2,2 t}^{2,1}} \leq 2 \sqrt{T} a_{o}\|g(t)\|_{L_{2}(0, T)}\|u-v\|_{B_{2,2, t}^{2,1}}
$$

in the sphere $\mathscr{K}_{0}$.

Consider the sequence $u_{k}(t, x)=Q\left(u_{k-1}(t, x)\right)$ in $B_{2,2, T}^{2,1}$ where $u_{o}(t, x)=0$. Using (3.1) and 
the inathematical induction we get for any $k(k=1,2,3, \ldots)$ and $t \in[0, T]$ :

$$
\begin{gathered}
\left\|u_{k}\right\|_{B_{2,2, t}^{2,1},}^{2} \leq 2\|W\|_{B_{2,2, T}^{2,1}}^{2}+8 T a_{o}^{2} \int_{0}^{t}\left\|F\left(u_{k-1}(\tau, x)\right)\right\|_{W_{2}^{1}(\Omega)}^{2} d \tau \\
\leq 2\|W\|_{B_{2,2, T}^{2,1}}^{2}+16 T a_{o}^{2}\left\{\int_{0}^{T} c^{2}(\tau) d \tau+\int_{0}^{t} d^{2}(\tau)\left\|u_{k-1}\right\|_{B_{2,2,1}^{2,1}}^{2} d \tau\right\} \\
=A^{2}+\int_{0}^{t} \mathscr{B}^{2}(\tau)\left\|u_{k-1}\right\|_{B_{2,2, t}^{2,1}}^{2} d \tau \\
\leq \mathcal{A}^{2}+\mathcal{A}^{2} \int_{0}^{t} \mathfrak{B}^{2}(\tau) d \tau+\ldots+\mathcal{A}^{2} \frac{\left\{\int_{0}^{t} \mathfrak{B}^{2}(\tau) d \tau\right\}}{(k-1) !}
\end{gathered}
$$

where

and

$$
\mathcal{A}^{2}=2\|W\|_{B_{2,2, T}^{2,1}}^{2}+16 T a_{o}^{2}\|c(t)\|_{L_{2}(0, T)}^{2}
$$

$$
\mathfrak{B}^{2}(t)=16 T a_{o}^{2} d^{2}(t)
$$

From (3.8) for any $k(k=1,2, \ldots)$, we get

$$
\left\|u_{k}\right\|_{B_{2,2, t}^{2,1}}^{2} \leq \mathcal{A}^{2} \cdot \exp \left\{\int_{0}^{T} \mathscr{B}^{2}(\tau) d \tau\right\}=C_{o}^{2}
$$

i.e., all $u_{k}(t, x)$ are contained in the sphere $\mathscr{K}_{o}$. Further, using (3.2) and (3.3) we get for any $t \in[0, T]$ and $k(k=1,2,3, \ldots)$

$$
\begin{aligned}
& \left\|u_{k+1}-u_{k}\right\|_{B_{2,2, t}^{2,1}}^{2} \leq 4 T a_{o}^{2}\left\|F\left(u_{k}(\tau, x)\right)-F\left(u_{k-1}(\tau, x)\right)\right\|_{L_{2}(\Omega)}^{2} d \tau \\
& \leq 4 T a_{o}^{2} \int_{0}^{t} g^{2}(\tau)\left\|u_{k}-u_{k-1}\right\|_{B_{2,2, t}^{2,1}}^{2} \\
& \leq\left\|u_{1}-u_{o}\right\|_{B_{2,2, T}^{2,1}}^{2} \frac{\left\{4 T a_{o}^{2} \int_{0}^{t} g^{2}(\tau) d \tau\right\}^{k}}{k !} \\
& =\left\|u_{1}\right\|_{B_{2,2}^{2,1}, T}^{\frac{\left\{4 T a_{o}^{2} \int_{0}^{t} g^{2}(\tau) d \tau\right\}^{k}}{k !}} \leq C_{o}^{2} \frac{\left\{4 T a_{o}^{2} \int_{0}^{t} g^{2}(\tau) d \tau\right\}^{k}}{k !}
\end{aligned}
$$

Therefore,

$$
\left\|u_{k+1}-u_{k}\right\|_{B_{2,2, T}^{2,1}}^{2} \leq C_{o}^{2} \frac{\left\{4 T a_{o}^{2}\|g(t)\|_{L_{2}(0, T)}^{2}\right\}^{k}}{k !},(k=1,2, \ldots)
$$


Then $\left\{u_{k}(t, x)\right\}$ is a fundamental sequence in $B_{2,2}^{2,1} T$. Since $B_{2,2, T}^{2,1}$ complete, then

$$
u_{k}(t, x) \quad B_{2,2}^{2,1}, T \quad u(t, x) \in \mathscr{G}_{0} \quad \text { as } k \rightarrow \infty
$$

Since $Q$ is continuous in $x_{o}$, then from the relation $u_{k}(t, x)=Q\left(u_{k}=1^{(t, x))}\right.$

we have

$$
u,(t, x)=Q(u(t, x))
$$

Therefore, as in (3.11), (3.12) the speed of convergence is governed by the following inequality

$$
\begin{gathered}
\left\|u_{k}-u\right\|_{B_{2,2}^{2,1}, T}^{2} \leq\left\|u_{o}-u\right\|_{B_{2,2, T}^{1,0}}^{2} \frac{\left\{4 T a_{o}^{2}\|g(t)\|_{L_{2}(0, T)}^{2}\right\}^{k}}{k !} \\
\leq C_{o}^{2} \frac{\left\{4 T a_{o}^{2}\|g(t)\|_{L_{2}(0, T)}^{2}\right\}^{k}}{k !},(k=1,2, \ldots) .
\end{gathered}
$$

Now to prove the uniqueness let us assume the $u(t, x)=\sum_{s=1}^{\infty} u_{s}(t) \ell_{2}(x)$ solution to $(2.1)-(2.3)$ then $F(u(t, x)) \in L_{2}\left(Q_{T}\right)$. By Lemma (1) $u_{s}(t)$ satisfy (2.9); from (2.9) we get

$$
\|u(t, x)\|_{B_{2,2, t}^{1,0} \leq} \leq\|W(t, x)\|_{B_{2,2, T}^{1,0}}+2 \sqrt{T}\|F(u(t, x))\|_{L_{2}\left(Q_{T}\right)}<+\infty
$$

Therefore $u \in B_{2,2, t}^{1,0}$. Since $u(t, x) \in W_{2}^{2}\left(Q_{T}\right) \cap B_{2,2, T}^{1,0}$ then by $(3.1) F(u(t, x)) \in W_{x, t, 2}^{1,0}\left(Q_{T}\right)$, but by condition 6 Theorem 2 for all $t \in[0, T], F(u(t, x)) \in \stackrel{\circ}{D}(\Omega)$. Thus using (2.9) with some manipulation

$$
\|u(t, x)\|_{B_{2,2, t}^{2,1}} \leq\|W(t, x)\|_{B_{2,2, T}^{2,1, T}}+2 \sqrt{T} a_{o}\|F(u(t, x))\|_{W_{x, t, 2}^{1,0}\left(Q_{T}\right)<+\infty}
$$

Therefore, $u \in B_{2,2, T}^{2,1}$. Then, using (3.1), (3.8), (3.10), we get $\|u(t, x)\|_{B_{2,2, t}^{2,1}} \leq C_{o}$. Thus, all almost everywhere solutions (2.1)-(2.3) belong to the sphere $K_{o}$ and they are fixed points in $B_{2,2}^{2,1}, T$ for operator $Q$. Le $u, v$ be two solutions to (2.1)-(2.3), then by (3.2) we get

$$
\begin{gathered}
\|u-v\|_{B_{2,2, t}^{2,1}}^{2} \leq 4 T a_{o}^{2} \int_{0}^{t} \| F(u(\tau, x))-F\left(\dot{v(\tau, x))} \|_{W_{2}^{1}(\Omega)}^{2} d \tau\right. \\
\leq 4 T a_{o}^{2} \int_{0}^{t} g^{2}(\tau)\|u-v\|_{B_{2,2, t}^{2,1}}^{2} d \tau
\end{gathered}
$$

Therefore, using Belmann's inequality [10] we have

$$
\begin{gathered}
\|u-v\|_{2,2, t}^{2}=0 \text { in }[0, T] . \text { Therefore, } u=v . \\
\underline{\text { REFERENCES }}
\end{gathered}
$$

\section{REFERENCES}

1. KRASNOSELSKII, M.A., "Topological methods in the theory of nonlinear integral equations." Pergamon Press, New York, 1964.

2. BURSKII, V.P., "Remarks on the kernel of a differential operator with constant coefficients in a domain.", Mat. Fix. Nelinein. Mekh. No. $\underline{2}, \underline{36}(1984), 43-45$. 
3. KAZARYAN, G.G., "Weak solutions of the Dirichlet problem for a quasilinear equation with lower-order terms." Trudy Math. Inst. Steklov. 170, (1984), 105-112.

4. KHALIFA, M.E., "The mixed problem of second order hyperbolic-parabolic system." Dokl. Akad. Nauk Az. USSR №. $\underline{5}$ (1979), 60-65.

5. KHALIFA, M.E., "The continuously dependent generalized solution for a mixed problem of the hyperbolic-parabolic system." Azerbaizan Gos. Univ. Ucen. Zap 3 (1979) 65-69.

6. KHALIFA, M.E., "Investigation of existence and uniqueness theorem for hyperbolic equation." Mans. Sci. Bull. Vol. 16(2).

7. LADYZENSKAJA, O.A., "Boundary value problems of mathematical physics." Nauk, Moscow, 1973.

8. LADYZENSKAJA, O.A., "The mixed problem for hyperbolic equations, Gostekhizdat, 1953.

9. SMIRNOV, V.I., "A course of Higer mathematics." Pergamon Press, New York, 1964.

10. BECKENBACH, E.F. and BELLMAN, R., Inequalities, Springer-Verlag, Berlin, 1961. 


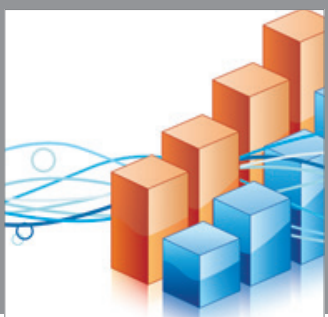

Advances in

Operations Research

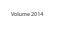

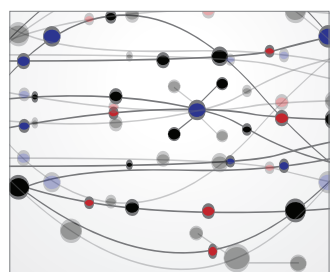

\section{The Scientific} World Journal
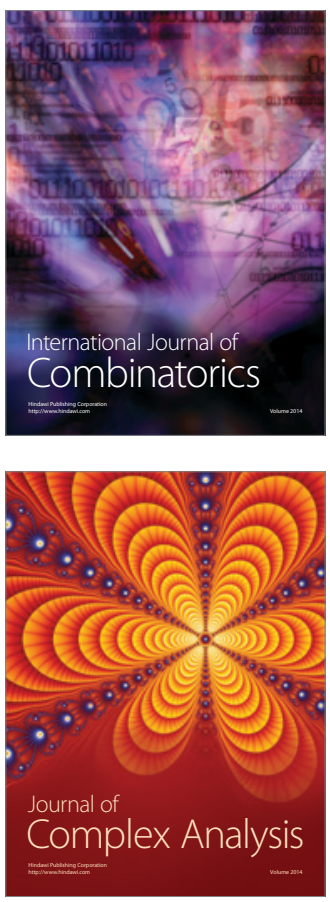

International Journal of

Mathematics and

Mathematical

Sciences
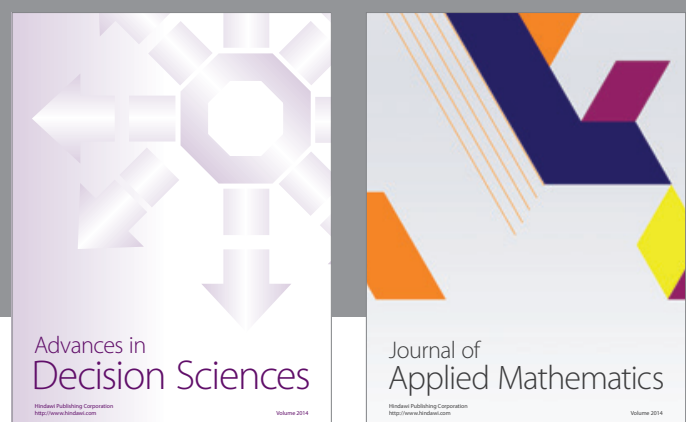

Journal of

Applied Mathematics
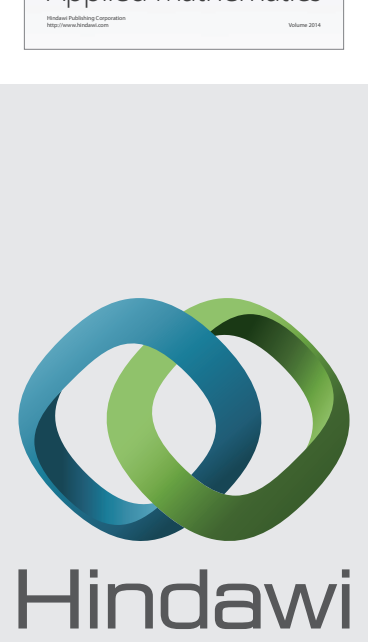

Submit your manuscripts at http://www.hindawi.com
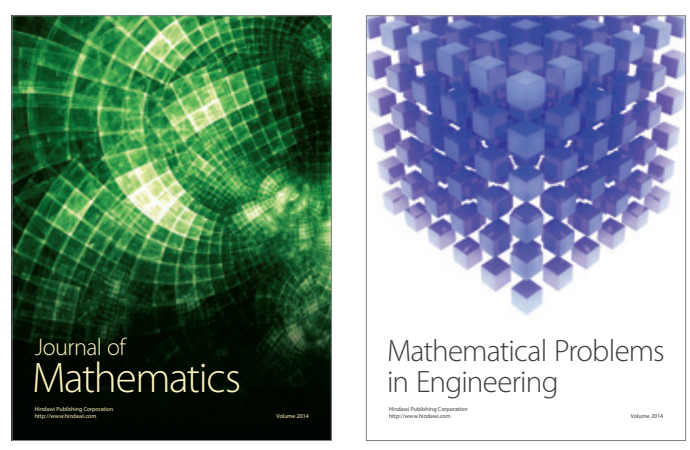

Mathematical Problems in Engineering
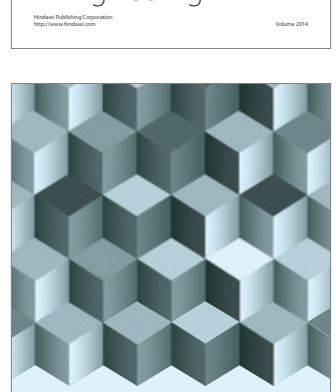

Journal of

Function Spaces
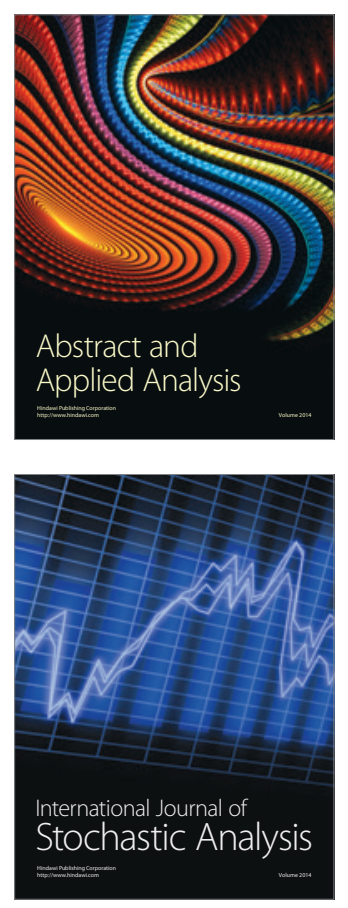

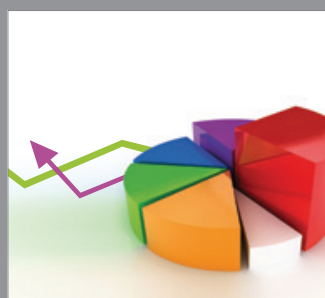

ournal of

Probability and Statistics

Promensencen
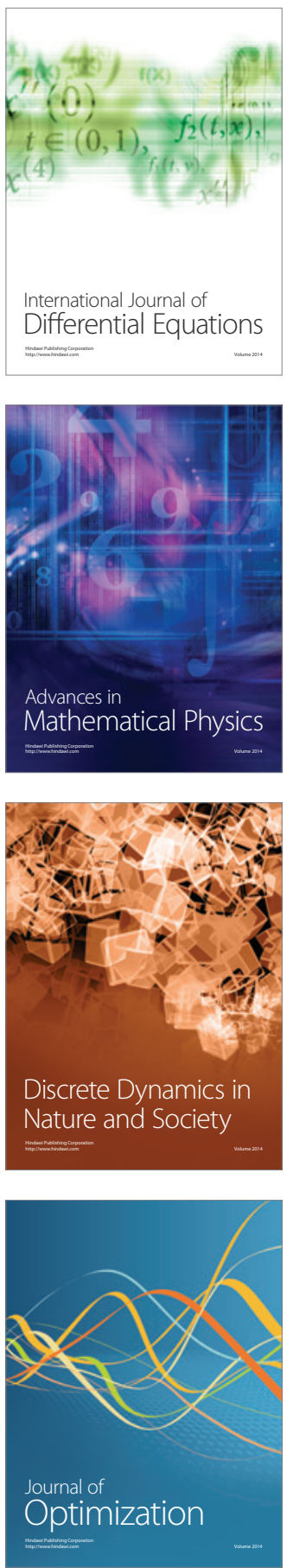